\title{
Estimations of the nonlinear properties of the internal wave field off the Israel coast
}

\author{
E. Pelinovsky ${ }^{1}$, T. Talipova ${ }^{2}$, and V. Ivanov ${ }^{3}$ \\ ' Technion, Haifa, Israel and Institute of Applied Physics, Nizhny Novgorod, Russia \\ ${ }^{2}$ Institute of Applied Physics, Nizhny Novgorod, Russia \\ ${ }^{3}$ Marine Hydrophysical Institute, Sebastopol, Ukraine
}

Received 27 May 1994 - Accepted 11 May 1995 - Communicated by V. Shrira

Abstract. The measurements of the vertical structure of hydrological fields and internal waves on the Levantine Sea's polygon in the Mediterranean, obtained in the 27 -th cruise of the RV "Professor Kolesnikov" in 1991, have been used to estimate the kinematic and nonlinear characteristics of the internal wave field. Statistical and spatial distributions of the vertical profiles of the BruntVaisala frequency are described. They have been used to calculate the coefficients of the Korteweg - de Vries equation. This equation forms the main model for nonlinear internal waves and their coefficients determine the speed of propagation of long waves, dispersive and nonlinear parameters. It is shown that the variations of the long wave speed propagation and the dispersion parameter are relatively small in comparison with the variation of the nonlinear parameter. Estimations of the nonlinear properties of the internal waves, being measured, based on the calculation of the local Ursell parameter are given. This method can be used for investigation of the internal wave transformation processes in oceanic regions with horizontal variability of the hydrophysical fields (temperature, salinity) and sloped sea floor.

\section{Introduction}

It is well known that the characteristics of the internal wave field, for example, its spectrum (the climatic spectrum, in terms of Garrett - Munk) and dispersion relation, depend on the vertical structure of the BruntVaisala frequency. The numerous efforts of classification of the Brunt-Vaisala frequency vertical profiles and study of their geographical distribution have been undertaken to predict internal waves in various regions of the World Ocean: the North Atlantic (Pereskokov, Shulepov, 1984), the North-West Pacific (Rostov et.al, 1988), the Black Sea (Blatov et.al, 1988; Ivanov et.al, 1994).

Nonlinear effects are essential to internal waves ow- ing to small speed of propagation, and nonlinear internal waves are observed very often (Ostrovsky, Stepanyants, 1989). The solitary waves in some regions of the World Ocean: the Middle Atlantic Bight (Zheng et.al, 1993), the Alboran Sea (Pierini, 1989), the Australian North West Shelf (Holloway, 1987), the Sulu Sea (Apel et.al, 1985), the Hudson Bay (Gan, Ingram, 1992), the Okhotsk Sea (Nagovitsyn et.al, 1991) were proven to be of the soliton nature. According to the theory of nonlinear wave processes, the evolution of arbitrary impulse disturbances results in solitons, and so they may contain a large part of the energy of internal waves. Thus, one appears to face the problem of predicting the possible parameters of the internal wave solitons in different regions of the World Ocean. To solve this problem, one can employ both the available data of hydrophysical information (temperature and salinity) over the region and the measured characteristics of the nonlinear internal waves.

The data of polygon measurements in the Mediterranean Sea (the Levantine Sea's polygon off the Israel Coast), obtained in the 27-th cruise of the RV "Professor Kolesnikov" (July - August, 1991) are used in the given paper for the estimation of kinematic and nonlinear properties of the internal wave motions. The analysis of hydrological data, measured in the polygon, is described in Section 2. These data are used to calculate the vertical profiles of the Brunt - Vaisala frequency, which determine many properties of the internal wave field. The theoretical model for the description of nonlinear internal waves is based on the well-known Korteweg de Vries equation, which is given in Section 3. Statistical estimations of the variability of the coefficients of the Korteweg - de Vries equation for the Levantine Sea's polygon are given in Section 4. It is found that the variations of the speed of long wave propagation and dispersion parameter are relatively small in comparison with the variation of the nonlinear parameter. Spatial distribution of kinematic characleristics of the internal wave 


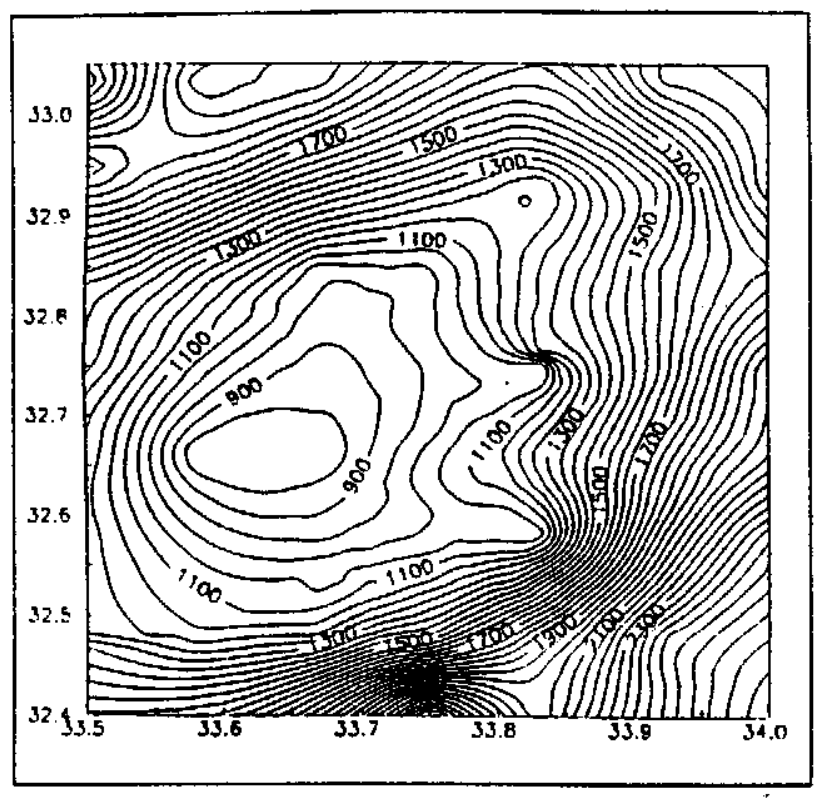

Fig. 1. The map of polygon in the Mediterranean.

field on the Levantine Sea's polygon is demonstrated in Section 5. Estimations of the nonlinear properties of the internal waves being measured, based on the calculation of the local Ursell parameter, are given in Section 6. These data can be used for the investigation of the internal wave transformation processes in this polygon and prediction of the wave characteristics in the parts of the polygon, where the measurements are absent.

2 Average Profiles of the Brunt - Vaisala Frequency and their Variations in the Levantine Sea

The map of the polygon in the Mediterranean (Levantine Sea's polygon) is shown in Fig. 1. The depths of the polygon are varied from $800 \mathrm{~m}$ to $2800 \mathrm{~m}$. There were 53 stations on the polygon in August 4-6, 1991. The vertical structure of temperature and salinity fields were measured by the hydrological complex MHI-4102 of the Marine Hydrophysical Institute (Sebastopol, Ukraine). The density of the sea water is defined by the known oceanographic formula through temperature, salinity and depth, and then the Brunt-Vaisala frequency $N(z)$ is calculated (Nikolaenko et al., 1992). All measurements were made only for depths up to $600 \mathrm{~m}$. Spatial distributions of the maxima $N(z)$ and their depth are shown in Figs. 2 and 3.

These data were used for the calculation of the mean vertical profile of the Brunt - Vaisala frequency and its rms variation. Since the depths of temperature and salinity measurements were different for the different stations (points), we have calculated the mean profile taking into account the various number of data on the same depths. The results of the calculation of the mean

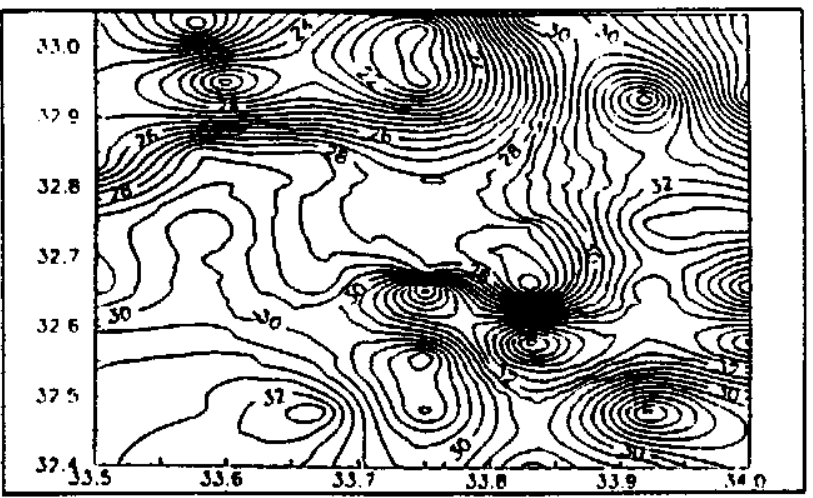

Fig. 2. Spatial distributions of maxima Brunt-Vaisala frequency.

profile $\bar{N}(z)$ and its rms deviation $\delta N(z)$ are shown in in Fig. 4 for depths less than $150 \mathrm{~m}$. One season pycnocline with a frequency 23 cycles/hour at the depth $25 \mathrm{~m}$ is selested in the average profile of the Brunt-Vaisala frequency. For a depth of more then $150 \mathrm{~m}$, this function can be described by the regression relation:

$$
\bar{N}(z)=4.64 \exp (-0,000416 h),
$$

where $N$ is in cycle/hour and $h$ is in $\mathrm{m}$. The curves of $\bar{N}(z)$ and $\delta N(z)$ are rather similar and large variations in the $N(z)$ are observed near the upper boundary of the pycnocline. The value of $\delta N / \bar{N}$ is large (90\%) at depths $15-20 \mathrm{~m}$ and constant $(15 \%)$ below the pycnocline (Fig. 5). Similar results have been obtained in our expedition in the Black Sea and at another region of the Mediterranean (Nikolaenko et.al, 1992). They are typical for the tideless seas in spring - summer hydrological season.

Polygon measurements of the Brunt-Vaisala frequency profiles for the Levantine Sea were fulfilled to give statistical and geographical characteristics of kinematic and nonlinear properties of internal waves and to establish the limits of the applicability of averaged models of wave motion which use only averaged (over large regions and times) profiles of the Brunt-Vaisala frequency.

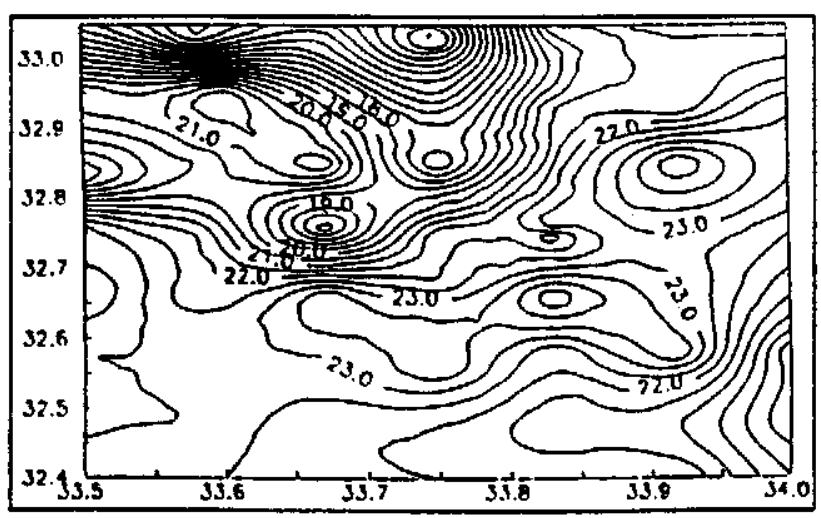

Fig. 3. Spatial distributions of the depth of maxima BruntVaisala frequency. 


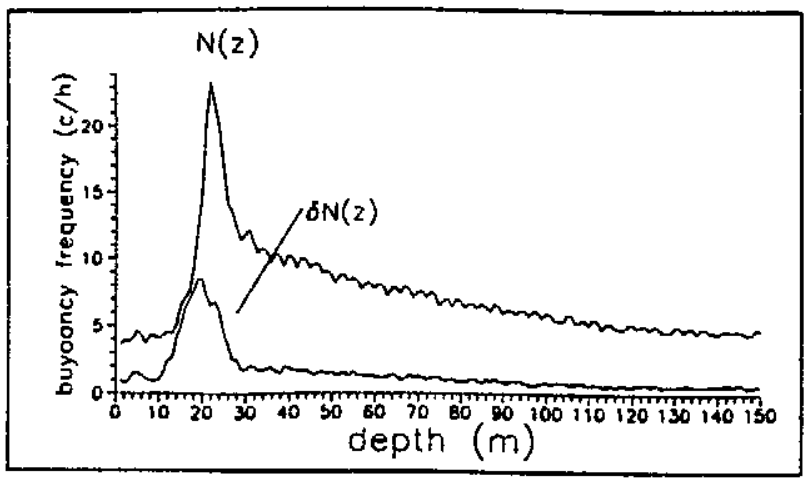

Fig. 4. Averaging profile of $N(z)$ and its rms deviation.

\section{Korteweg - de Vries Equation for Internal Wave Field}

Usually, internal waves of large energy have wavelengths of the order of $1000 \mathrm{~m}$ or more. For such long waves, the Korteweg - de Vries equation is an appropriate physical model for the description of the nonlinear and dispersive properties of internal wave field (Benney, 1966; Lee \& Beardsley, 1974; Pelinovsky et.al, 1977; Grimshaw, 1981, 1983; Gear \& Grimshaw, 1983; Smyth, Holloway, 1988; Zhou \& Grimshaw, 1989). It has the following form:

$$
\frac{\partial \eta}{\partial t}+c \frac{\partial \eta}{\partial x}+\alpha \eta \frac{\partial \eta}{\partial x}+\beta \frac{\partial^{3} \eta}{\partial x^{3}}=0
$$

where $\eta(x, t)$ is the displacement of the pycnocline, $x$ is a horizontal coordinate, $t$ is time and parameters $\alpha$ and $\beta$ are determined by the hydrology of the region:

$$
\alpha=\frac{3 c}{2} \frac{\int_{-H}^{0} \rho_{0}\left[\frac{d W}{d z}\right]^{3} d z}{\int_{-H}^{0} \rho_{0}\left[\frac{d W}{d z}\right]^{2} d z}, \quad \beta=\frac{c}{2} \frac{\int_{-H}^{0} \rho_{0} W^{2} d z}{\int_{-H}^{0} \rho_{0}\left[\frac{d W}{d z}\right]^{2} d z},
$$

where $\rho$ is the sea density. The phase speed of linear long waves $c$ and the structure of vertical displacement of the pycnocline in mode $W$ are determined by the eigenvalue

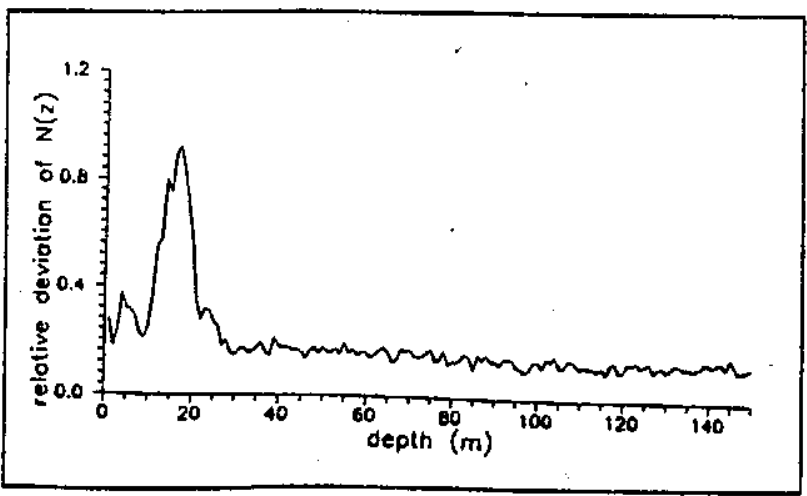

Fig. 5. Relative deviation of $N(z)$ problem:

$$
\frac{d^{2} W}{d z^{2}}+\frac{N^{2}(z)}{c^{2}} W=0, \quad W(-H)=W(0)=0,
$$

$W(z)$ is normalized by its maximal value. It means that $\eta(x, t)$ is the isopycnal surface with maximal displacement.

Solitary waves (solitons) are the steady-state solutions of the Korteweg - de Vries equation:

$$
\eta=a \cosh ^{-2} \frac{x-V t}{\Delta},
$$

where the soliton velocity $V$ and its half-width $\Delta$ are determined by the wave amplitude $a$ :

$$
V=c+\frac{\alpha a}{3}, \quad \Delta=\left(\frac{12 \beta}{\alpha a}\right)^{1 / 2} .
$$

Although the solution (5) and its properties are concerned with an arbitrary wave mode, only the first mode is discussed below, because it dominates, as a rule.

It should be emphasized that the dispersion parameter $\beta$ is always positive, but the nonlinear parameter $\alpha$ can be either negative or positive (or zero). In the approximation under consideration, solitons can exist only when $\alpha \neq 0$ (in the opposite case it is necessary to take into account the cubic nonlinearity). Besides the product, $\alpha a$ is always greater than 0 , so $a>0$ when the pycnocline is displaced upwards, in the opposite case $a<0$.

The Korteweg - de Vries equation is completely integrable (see, for example, Newell, 1985), anf this fact enables us to consider the nonstationary processes for arbitrary time. The evolution of arbitrary disturbance depends on the parameter:

$$
U=|H| L^{2},
$$

where $H$ is a height and $L$ is a half-width of initial disturbance. This parameter is proportional to the Ursell parameter in the theory of surface waves. In particular, if $U<U_{c}$, where

$$
U_{c}=\frac{12 \beta}{|\alpha|}
$$

the evolution of an initial disturbance results in only one soliton (provided it has the proper polarity) and a large oscillatory tail. If $U>U_{c}$, then several solitons will be generated. The parameter $U$ can be treated as a criterion for nonlinearity of the wave motion. One can calculate the parameter $U$ for the available internal wave field records to determine their nonlinearity. Such an approach has been used in (Serebryany, 1990; Goryachkin et al, 1992) for the analysis of real records of the internal waves using a simplification of the ocean stratification. For some density profiles the coefficients of 
Korteweg - de Vries equation can be obtained in an explicit form. For example, if the sea stratification in the upper layer can be described as a two-layer model with a density jump $\Delta \rho$ on the depth $h$, the coefficients of Korteweg - de Vries equation are found in an algebraic form

$$
\begin{gathered}
c=\sqrt{\frac{g \Delta \rho h(H-h)}{\rho H}}, \\
\alpha=\frac{3 c\left[h^{2}-(H-h)^{2}\right]}{2 H h(H-h)} \\
\beta=\frac{c h(H-h)}{6} .
\end{gathered}
$$

It allows us to write the analytical formula for soliton parameters as

$$
\begin{gathered}
\Delta=\left[\frac{4 H h^{2}(H-h)^{2}}{3\left[h^{2}-(H-h)^{2}\right] a}\right]^{1 / 2}, \\
U_{c}=\frac{4 H h^{2}(H-h)^{2}}{3\left\{\left[h^{2}-(H-h)^{2}\right] \mid\right.},
\end{gathered}
$$

The next limiting case of sea stratification is the sea of a very large depth when the influence of the season pycnocline can be neglected and the Brunt - Vaisala frequency can be considered as a constant in depth. In this case the calculating formula in Boussinesq approximation is as follows:

$$
c=\frac{N H}{\pi}, \quad \beta=\frac{N H^{3}}{2 \pi^{2}}, \quad \alpha=0 .
$$

In the framework of the constant Brunt - Vaisala frequency model and the Boussinesq approximation, the quadratic nonlinearity is absent and the long waves with scales greater than the sea depth become linear. In fact, we have to take into account the terms of the next order in nonlinearity and do not have to use the Boussinesq approximation. In this case we obtain the modified Korteweg - de Vries equation. Thus it is clear that the nonlinear effects are smaller in this case.

As it follows from the asymptotic expressions given above, the nonlinear parameter $\alpha$ can vary nonmonotonically with depth. It is positive at small depths (the pycnocline is close to the bottom), equal to zero when the pycnocline lies at the depth $H / 2$ and increases with its absolute value, being negative at a definite depth, and tends to zero at large depths. Such asymptotic expressions, of course, take place in the limiting cases which turn out to be impossible in real situations. Therefore it is necessary to make special numerical calculations for regions with real stratification based on the formula (3) - (8) Making such calculations for the Levantine Sea's polygon, we have added the absent data of BruntVaisala frequency for depths greater than $600 \mathrm{~m}$ by the commonly used exponential approximation:

$$
N(h)=N(600 m) \exp \{0.000416(600-h)\}
$$

in accordance with Eq. (1).

A few words about the applicability of this theoretical model associated with the Korteweg - de Vries equation. First, we did not take into account the currents that usually are present in the ocean. However, as it is seen from the calculations, the speed of long wave propagation appears to be equal to $2-5 \mathrm{~m} / \mathrm{sec}$, and, therefore, the currents with typical velocities $50 \mathrm{~cm} / \mathrm{sec}$ (unfortunately, the data on the currents in this region are unavailable to us) will not be essential for the kinematic characteristics of long waves. Second, we did not take into account the dispersion due to the Earth's rotation. In the long wave approximation the dispersion relation for internal waves is given by the well - known equation (Shrira, 1986; Ostrovsky, Stepanyants, 1990)

$$
\omega^{2}=f^{2}+c^{2} k^{2}\left(1-2 \beta k^{2}\right)
$$

where $f$ is a Coriolis parameter. It is seen that in the case $2 \beta c^{2} k^{4} \gg f^{2}$ we can neglect the dispersion due to the Earth's rotation, and this condition is satisfied for waves of lengths $1-10 \mathrm{~km}$. Third, Eq. (2) does not include the terms responsible for horizontal variability of the parameters of the ocean. Analysis carried out in Sections 4 and 5 shown the strong variability of all coefficients of the Korteweg - de Vries equation which influence on on the variability of the nonlinear internal wave field. Because the horizontal variability of the characteristics of the ocean medium is enough smooth we can ignor the reflection of the wave energy from horizontal inhomogeneties and seek the solution for the vertical displacement of the pycnocline in a form: $\eta(t, \vec{r}) W(z, \epsilon \vec{r})$, where $W$ is again the vertical structure of the pycnocline displacement, found from Eq. (4), and the small parameter $\epsilon$ characterizes the smoothness of the horizontal inhomogenety of the ocean medium. Corresponding equation, obtained with using of the asymptotic procedure, can be written in following form (Pelinovsky et al, 1977; Zhou \& Grimshaw, 1989):

$$
\frac{\partial \eta}{\partial l}+\frac{\alpha \eta}{c^{2}} \frac{\partial \eta}{\partial s}+\frac{\beta}{c^{4}} \frac{\partial^{3} \eta}{\partial s^{3}}+\frac{1}{2 Q J} \frac{d Q J}{d l} \eta=0,
$$

where

$$
s=\int \frac{d l}{c}-t
$$

is a time in the system of coordinates moving with a linear speed of long waves, $l$ is the distance along the ray which is found from eikonal equation

$$
(\nabla \tau)^{2}=c^{-2}(\vec{r})
$$

$J$ is a differential width of a ray beam, and

$$
Q=\frac{c^{3} \int_{-H}^{0}(d W / d z)^{2} d z}{c_{0}^{3} \int_{-H}^{0}\left(d W_{0} / d z\right)^{2} d z}
$$

and values with index " 0 " are the values at any initial point $l_{0}$. Additional term in Eq. (15) depends on the 
wave geometry (the curvature of wave fronts). We have no measured data about the spatial distribution of the internal wave field and, as a first step, we will study here only characteristics of nonlinear internal waves related with the density stratification and bottom bathymetry. These characteristics can be considered in the frame of the Korteweg - de Vries equation (2).

\section{Statistical Estimations of the Korteweg - de Vries Coefficients}

We have used the following procedure for statistical estimations of the Korteweg - de Vries equation coefficients (this method has been developed for any polygon measurements in (Ivanov et.al, 1992)):

- The Korteweg - de Vries equation coefficients $c, \alpha, \beta$ as the functions of depth have been calculated with the use of the average vertical profile of the Brunt-Vaisala frequency $\bar{N}(z)$.

- The same calculations have also been made by using two vertical profiles of the Brunt-Vaisala frequency $\bar{N}(z) \pm \delta N(z)$, which characterize the deviations of the hydrological fields This procedure allows us to obtain the deviations of the Korteweg - de Vries equation coefficients.

- Similar calculations, by using the data of the real stations in the Levantine Sea's polygon, have been made to estimate the applicability of average dependencies.

The results of calculations, having been obtained by this method, are shown here. Figs $6-8$ show the linear speed of wave propagation $c$, parameters of dispersion $\beta$ and nonlinearity $\alpha$ as functions of depth for different cases of the numerical solution of the eigenvalue problem (4): for profiles $\bar{N}(z)$ (solid lines) and $\bar{N}(z) \pm \delta N(z)$ (dashed lines with \pm ). Here the data of calculations for the real stations in the polygon are also given. The character of these curves confirms the ideas mentioned above concerning the dominant role of a season pycnocline at small depths and the possibility of using a two-layer model in this case. For large depths, the approximation of exponentially stratified sea with the mean Brunt - Vaisala frequency $N=4$ cycle $/ \mathrm{hr}$ ( 1 cycle $/ \mathrm{hr}=210^{-3} 1 / \mathrm{sec}$ ) is preferred for the calculation of $c$ and $\beta$. As it is seen, the values of propagation speed and dispersion, calculated on the basis of the data of real hydrological stations in the polygon are in good agreement with those calculated by using the mean Vaisala frequency. Therefore, one may use the data of a single vertical temperature and salinity profile and the bathymetric map to estimate, in the first (rough) approximation, the values of the kinematic characteristics of linear internal waves on the polygon. But the accuracy of such calculations is rather poor and the error may reach $40 \%$. It can be easily explained within the model of exponential density stratification, where $c$ and $\beta$ are proportional to the Brunt - Vaisala frequency, such model is valid at

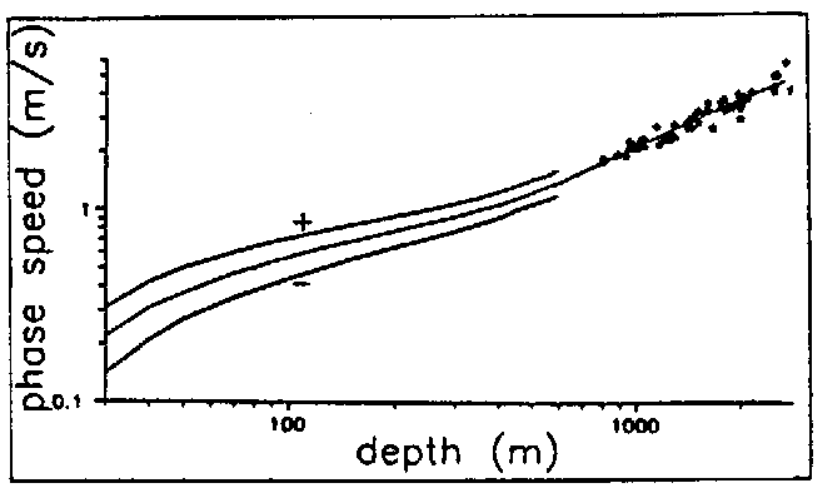

Fig. 6. Linear wave speed: calculations for $\hat{N}(z)$ (solid lines) and $\hat{N}(z) \pm \delta N(z)$ (dashed lines with \pm ). Points are data of calculations for the real stations in the polygon.

the large depths (see 12) and, consequently, the error is $2 \delta N / \bar{N}=40 \%$.

It is interesting to note the nonmonotonous character of the nonlinear parameter dependence on depth (Fig. 8). The results of calculations on the basis of real station measurements yield large deviations, by more than one order. It is related to the infuence of the main layer of the sea (below the pycnocline) where the stratification may be approximated by an exponential law, for which the nonlinearity parameter is absent in compliance with (12). Evidently, the relative variations of nonlinearity (when its mean value is small) are not small and the use of calculations based on the mean values is not effective.

The obtained values of the Korteweg - de Vries equation coefficients have been used for calculation of the "soliton" Ursell parameter by Eq. (8). The limits of deviations of this parameter are defined by

$$
U_{+}=\frac{12 \beta_{+}}{\alpha_{-}}, \quad U_{-}=\frac{12 \beta_{-}}{\alpha_{+}} .
$$

The results of the calculations of the averaged $U_{c}$ and its variations are shown in Fig. 9. As it is seen, the values of the Ursell parameter are large at large depths

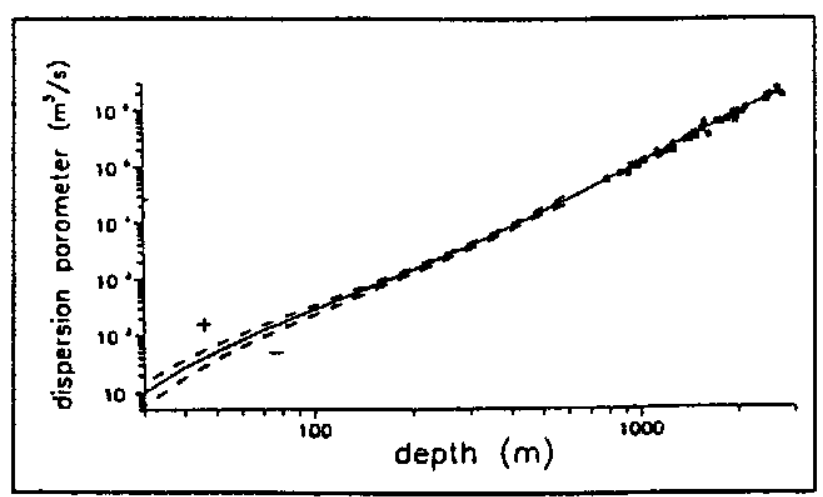

Fig. 7. Dispersion parameter: calculations for $N(z)$ (colid lines) and $\bar{N}(z) \pm \delta N(z)$ (dashed lines with \pm ). Points are dats of calculations for the real stations in the polygon. 


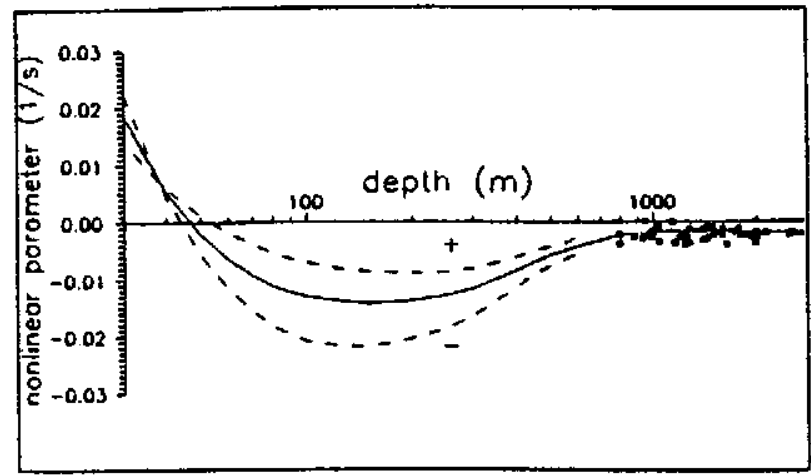

Fig. 8. Nonlinear parameter: calculations for $N(z)$ (solid lines) and $\bar{N}(z) \pm \delta N(z)$ (dashed lines with \pm ). Points are data of calculations for the real stations in the polygon.

and in the range of depths $40-50 \mathrm{~m}$, where the nonlinearity parameter is small. Here the waves must be more linear than for other depths. The Ursell parameter reaches its minimum at the depth about $60 \mathrm{~m}$ (and, of course, at depths less than $30 \mathrm{~m}$, which are not considered here) and it is equal to $10^{5} \mathrm{~m}^{3}$. In fact, it may be confirmed when the depth is greater than $40 \mathrm{~m}$ and wave parameters (amplitude and wavelength) are such that $U<10^{5} \mathrm{~m}^{3}$, then the nonlinearity of internal waves may be neglected (in this case the actual soliton formation is also possible but only as a single one and its amplitude is less than the initial value (Newell, 1985)). If the wave parameters are such that $U>10^{5} \mathrm{~m}^{3}$, the result will depend on the sea depth; thus to confirm the nonlinearity of the wave field, it is necessary to compare the local Ursell parameter defined by (7) with its "soliton" value $U_{c}$ depending on the sea depth.

The deviation of the Ursell parameter in terms of Eq. (19), taking into account the assumption of the statistical independence of $\alpha$ and $\beta$, is approximately $80 \%$. For examining the real deviation, we have repeatedly used the results of calculation of the Korteweg - de Vries equation coefficients for real stations on the polygon. They are shown by appropriate points in Fig. 9. It is seen that such calculations offer wider deviations for

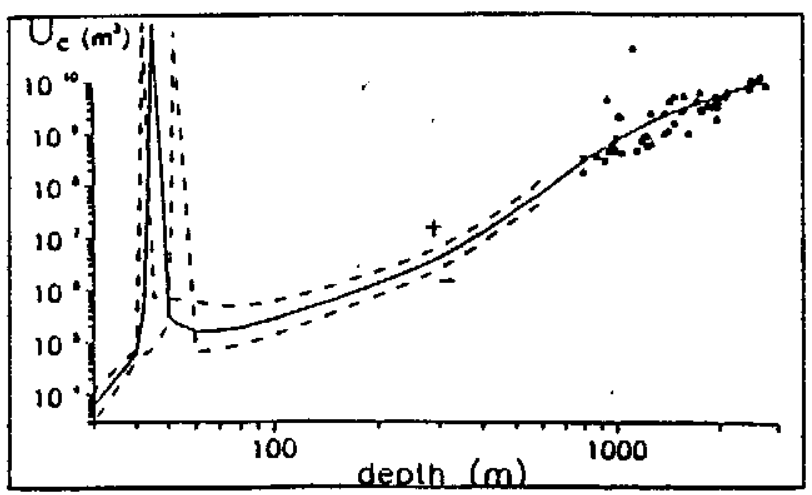

Fig. 9. Averaging profile of $U_{c}$ and its rms deviation. Points are data of calculations for the real stations in the polygon. depths near $100 \mathrm{~m}$ (more than two orders), as explained by the respective deviations of nonlinear parameter $\alpha$.

Therefore, for a rough estimation of the linematic characteristics of linear internal waves in a large polygon, it is enough to use the vertical profile of density (or temperature and salinity) and the map of depths on the polygon. But this approximation is too rough for estimation of the nonlinear properties of the internal wave field.

\section{Spatial Distribution of the Korteweg - de} Vries Equation Coefficients

It has been shown above that the approximation of the mean Brunt - Vaisala frequency is sufficiently rough for calculation of the Korteweg - de Vries equation coefficients, and, therefore, for estimation of the nonlinear properties of internal waves. Under these conditions, calculation of the wave characteristics can be made on the basis of real spatial distribution of the parameters mentioned above, as in the similar problems of long surface waves (tides, tsunami waves), where the bathymetric maps are used. Since the internal wave characteristics depend on bathymetry and hydrology, the maps of the Korteweg - de Vries equation coefficients play the same role as the bathymetric map for surface waves. isolines of the Korteweg - de Vries equation coefficients are shown in Figs. 10-12. It is interesting to compare the calculated maps with the bathymetry one (Fig. 1) and the maps of Brunt - Vaisala frequency (Figs. 2 and 3 ). As it was expected, spatial distributions of the propagation speed $c$ and dispersive parameter $\beta$ roughly correlate with the bottom bathymetry in case of large variations of depth. In particular, an underwater hill with coordinates $33.55-33.65^{\circ} \mathrm{E}, 32.50-32.77^{\circ} \mathrm{N}$ is manifested as a decrease of propagation speed and dispersive parameter in this region. In this area smaller inhomogeneities of depth may be compensated by inhomogeneities of the Brunt - Vaisala frequency and furthermore, they are not manifested on the maps (Figs. 10 and 11). The nonlinear parameter $\alpha$, which has been shown, depends on both depth and Brunt - Vaisala frequency variations. In addition, there are many small scale regions in a corresponding map to compare with initial maps (Fig. 12).

The maps of the Korteweg - de Vries equation coeffcients permit one to calculate the evolution of the wave field at a long distance, taking into account its nonlinearity and dispersion. Since the scales of variability of the Korteweg - de Vries equation coefficients are much more than the typical lengths of the internal waves, (several kilometers) this equation may be seen as an equation with constant coefficients at small distances and consequently, the well-known theory of soliton formation from impulse perturbations (or the recurrence) can be applied to the description of internal waves. Further- 


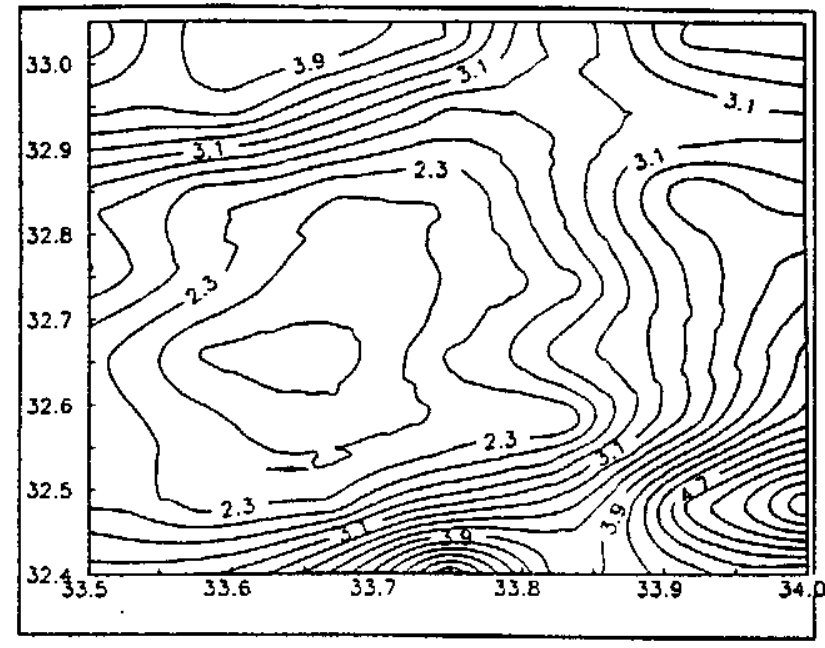

Fig. 10. Spatial distribution of linear wave speed.

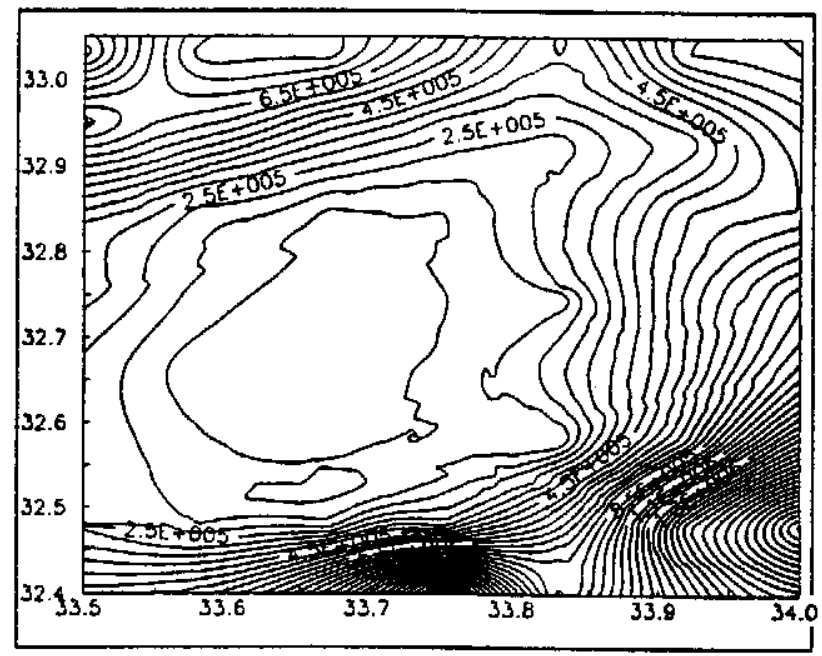

Fig. 11. Spatial clistribution of dispersion parameter.

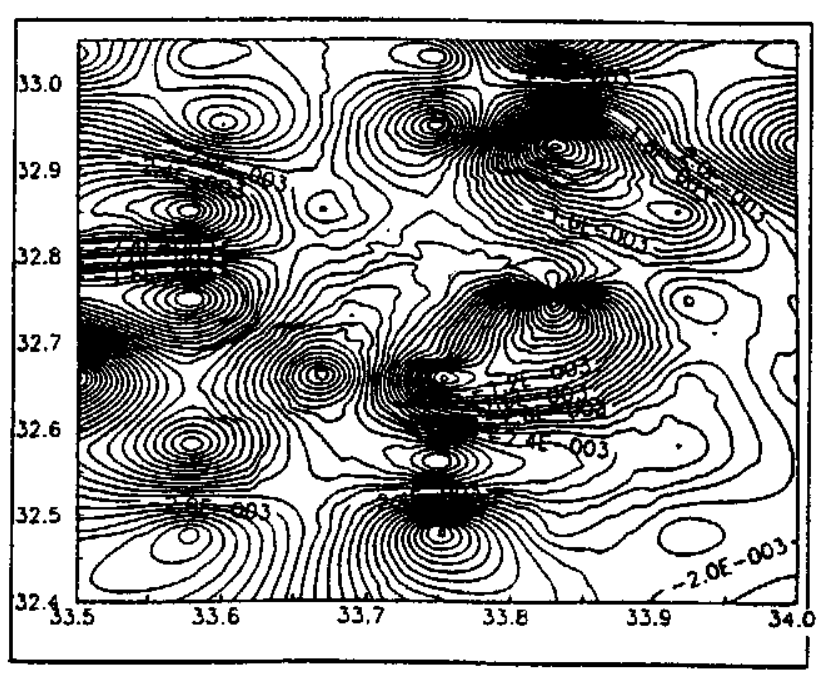

Fig. 12. Spatial distribution of nonlinear parameter. more, the coefficients are varied slowly, the use of ray methods for studying the internal wave transformation in a sea with horizontal inhomogeneities of depth and density field is possible (some analytical solutions and examples of numerical simulation for certain models are given in (Pelinovsky \& Shavratsky, 1976; Smyth \& Holloway, 1988; Zhou \& Grimshaw, 1989; Pelinovsky et.al, 1994). We suppose to consider this problem in the future, but some points that follow from the ray methods, should be mentioned here. In particular, the regions of decrease of the propagation speed play the role of underwater mountains for surface waves concentrating their energy there. Besides, the underwater mountains can trap part of wave energy, and as a result, the resonance peaks may appear at the fixed frequencies. These significant problems, which are well known for surface waves, have not been considered for internal waves yet, especially for the real horizontal variability of sea hydrology.

\section{The Estimations of Nonlinear Properties of Internal Waves, Measured on the Polygon}

We attempt at using this theory for rough estimation of the nonlinear properties of internal waves measured in the Levantine Sea's polygon. During 27 - 29 July, 1991, in the 27-th cruise of the RV "Professor Kolesnikov", an experiment on recording internal waves was performed. It consisted in towing of the MHI- 4106 probe (a distributed temperature sensor of length $25 \mathrm{~m}$ ) in the thermocline. The description of the measurement data is given in (Ivanov et.al, 1993). The parameters of seven intern al waves with relative large amplitudes are given in Table 1. The values of the Ursell parameters calculated for these waves have the order of $(2.1-5.3) 10^{6} \mathrm{~m}^{3}$ that is much more than the minimum value of "soliton" Ursell parameter $\left(10^{6} \mathrm{~m}^{3}\right)$. The upper and lower limits of the calculated Ursell parameters are shown as horizontal dashed lines in the Fig. 13, and the curve $U_{c}(H)$ is also given. As it can be seen, all calculated values of the Ursell parameter are less than the "soliton" curve for depths more than $350 \mathrm{~m}$. Therefore, it can be said that all measured internal waves may be treated as almost linear. But when the wave reaches shallow water (less than $300 \mathrm{~m}$ ), it becomes nonlinear. Furthermore, when the depth becomes less than $200 \mathrm{~m}$, the exceeding of the Ursell parameter over the "soliton" value is so large that, from the point of view of the nonlinear wave theory, the dispersion is generally "put off" and does not influence the subsequent wave evolution. As a result, the wave will transform to a shock (bore) under the action of the nonlinearity and small dispersion induces many oscillations (solitons) behind the shock front. It is simple to define the form of this wave and the number of solitons (see, for example, Newell, 1985). These theoretical predictions for the waves in the Levantine Sea are 
Table 1. Characteristics of measured internal waves

\begin{tabular}{cll}
\hline Height $(\mathrm{m})$ & Wavelength $(\mathrm{km})$ & Ursell Parameter $\left(10^{6} \mathrm{~m}^{9}\right)$ \\
\hline 1.5 & 5.5 & 11.3 \\
1.6 & 5 & 10.0 \\
1.7 & 5 & 10.6 \\
2.7 & 2.6 & 4.6 \\
1.2 & 2.6 & 2.0 \\
1.2 & 2.9 & 2.5 \\
1.8 & 2.8 & 3.3 \\
\hline
\end{tabular}

correlated with the well-known facts of strong deformation of internal waves on the shelves. In particular, they were observed in tidal waves on the Guinean shelf during the 20-th cruise of RV "Professor Kolesnikov" (Goryachkin et al, 1992). We could not obtain experimental results about the internal wave transformation on the shelf of the Mediterranean Sea. Nevertheless we can predict strong nonlinear deformation of internal waves on the shelf, using theoretical results mentioned above. For more accurate estimations, it is absolutely necessary to study the processes of internal wave transformation from the Levantine Sea to the shelf, and the calculated maps of the Korteweg - de Vries equation coefficients can be useful for numerical simulations. It may be the topic of the further theoretical and experimental works.

\section{Conclusions}

The statistical and spatial distributions of the kinematic and nonlinear properties of the internal wave field in the Levantine Sea's polygon in the Mediterranean have been investigated. This analysis is based on the measurements of the vertical structure of the hydrological fields and internal waves obtained in the 27-th cruise of the RV "Professor Kolesnikov" in 1991. The coefficients of the Korteweg - de Vries equation, describing the nonlinear internal waves have been calculated within the averag-

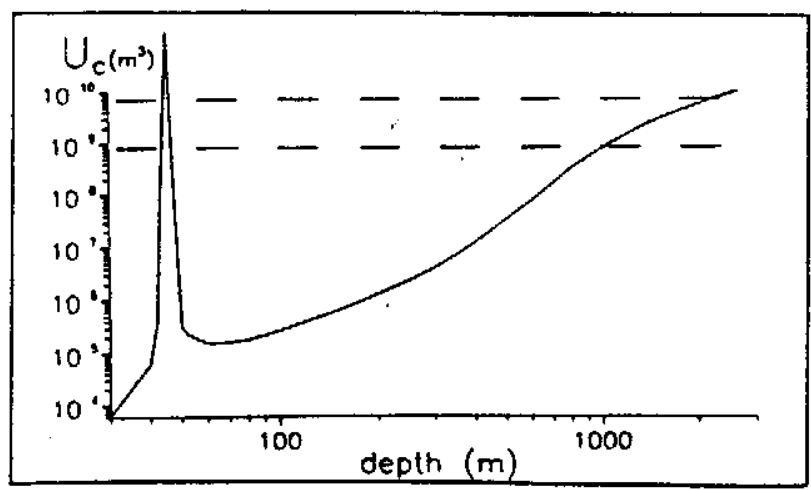

Fig. 13. Ursell parameter. Solid line corresponds to $U_{c}$. The upper and lower limits of calculated $U$ for measured waves are shown as horizontal dashed lines. ing models of density stratification, using data of real hydrological stations. It is shown that the variations of long wave speed propagation and dispersion parameter are relatively small, in comparison with the variation of the nonlinear parameter. Such characteristics can be calculated using the data of one hydrological station and the bathymetric map of the polygon. For calculation of the nonlinear parameter, it is important to produce the data from all hydrological stations in the polygon. The nonmonotonical character of the nonlinear parameter, changing with depth, is obtained. The estimations of the nonlinear properties of measured internal waves are based on the calculation of the local Ursell parameter. Its strong dependence on depth results in the broadcasting processes of breaking of the internal waves as "plunging" with the formation of a wave bore. The developed method can be used for investigation of the processes of internal wave transformation in oceanic regions with horizontal variability of hydrophysical fields.

Acknowledgements. This work was supported by grants from the ISF (NOSOOO), RFBR (93 - 05 - 8073), and the Technion Fund. We are grateful to V.Shrira for his help in the publication of this paper and useful discussion and comments. We are also indebted to A. Kraev for assistance in translation the manuscript from Russian. EP would like to thank M.Stiassnie and Y.Agnon for warm hospitality during his visit to Israel.

\section{References}

Apel, J. R. et al., The Sulu Sea international soliton experiment, J. Phys. Oceanogr., 15, 1625 - 1651, 1985.

Benney, D. J., Long nonlinear wave in fluid flows, J. Math. Phys, 45, 52 - 63, 1966.

Blatov, A., Ivanov, V., and Rossov, V., Physical and geographical features of Black Sea wave processes, in: Black Sea's Hydrophysical and Hydrochemical Field Forming end Year's Vari. ability Protesses (Ed. Eremeev V.). Sebastopol: Marine Hydrophysical Institute, 51 - 59, 1988, (in Russian).

Gan, J., and Ingram, R. G., Internal hydraulics, solitons and associated mixing in a Stratified Sound, J. Geophys. Res., C97, 9669 - 9688, 1992.

Gear, J. A., and Grimshaw, R., A second order theory for Bolitary waves in shallow fluids, Phys. Fluids, 26, 14-29, 1983.

Goryachkin, Yu., Ivanov, V., and Pelinovsky, E., Transformation of internal tidal waves over the Guinean Shelf, Sov. J. Phys. Oceanogr., s, 309 - 315, 1992.

Grimshaw, R., Evolution equations for nonlinear internal waves in stratified shear flows, Stud. A ppl. Math.,65, 159-188, 1981.

Grimshaw, R., Solitary waves in density stratified fluids, in: Nonlinear Deformation Waves. Springer, Berlin, 432 - 447, 1983.

Holloway, P. E, Internal hydraulic jumps and solitons at a Shelf Break Region on the Australian North West Shelf, J. Geophys. Res., C92, 5405 - 5416, 1987.

Ivanov, V., Pelinovsky, E., Stepanjants, Yu., and Taljpova, T., Statistical estimations of nonlinear long wave parameters at polygon measuring, Izvestija, Atmospheric and Oceanic Physics,28, 1062 - 1070, 1992.

Ivanov, V., Pelinovsky, E., and Talipova, T., Frequency of internal wave amplitude recurrence in the Mediterranean Sea, Oceanol. ogy, $99,180-185,1993$. 
Ivanov, V., Pelinovaky, E., Talipova, T., and Troitskaya, Yu., Geogrophical estimations of parameters of nonlinear internal waves on the polygon "South Crimea Beach" of the Black Sea, Morskoi Gidrof. Zh. (Marine Hydrophys. J.),2, $10-15,1994$, (in Russian).

Lee, C. H., and Beardnley, Y., The generation of long nonlinear internal waves in a weakly stratified shear flow, J. Geophys. Res., 79, 453 - 462, 1974.

Nagovitsyn, A., Pelinovsky, E., and Stepanyants, Yu., Obeervotion and analysis of solitary internal waves in the cosstal zone of the Sea of Olkhotsk, Sov. J. Phys. Oceanogr., 2, 65-70, 1991.

Newell, A., Solitons in Mathematico and Physics. Soc. Ind. Appl. Math., Philedelphia, 1985.

Nikolaenko, E., Pelinovsky, E., and Talipova, T., The averaging profiles of Brunt - Vajasala frequency and its variation in tideless seas in spring-summer hydrological season, in: $\mathrm{Hy}$ drophysical and Hydrochemical Investigations of Black Sea (Ed. V.Eremeev). Sebastopol: Marine Hydrophysical Institute, 80 91, 1992 (in Ruasian).

Ostrovsky, L. A., and Stepanyants, Yu. A., Do internal solitons exist in the ocean? Reviewe of Geophysics,27, $293-310_{2} 1989$.

Ostrovsky, L. A., and Stepanyants, Yu. A., Nonlinear surface and internal waves in rotating fluids, in: Nonlinear Waves (Eds. Gaponov - Grekhov A.V., Rabinovich M.I., Engelbrecht J.). V. 3, Springer - Verlag, $106-128,1990$.

Pelinovaky, E., and Shavratsky, S., Propagation of nonlinear internal waves in an inhomogeneous ocean, Izvestiya, Atmospheric and Oceanic Physics, 12, 41 - 44, 1976.

Pelinovaky, E., Shavratsky, S., and Raevaky, M., The Korteweg - de Vries equation for nonstationary internal waves in an inhomogeneous Ocean, Jzvestiya, Atmospheric and Oceanic Physics,19, 226 - 228, 1977.
Pelinovaky, E., Talipova, T., and Stepanyante, Yu., Modeling of nonlinear internal wave propagation in the horizontally inhomogeneaus ocean, Izvestiya, Atmospheric and Oceanic Physics,s8, $79-85,1994$.

Pereshkokov, A. I., and Shulepov, V. A., Density atratificstion and disperaion relations for internal waves in the North Atlantic, Oceenology, 24, 750 - 755. 1984.

Pierini, S., A model for the Alboran Sea internal nolitary waves, J. Phys. Oceenogr.,19, 755 - 772, 1989.

Rostov, I., Gladyshev, S., and Novotrjasov, V., Eatimations of internal wave parameter posible values for various wes water stratification of North-West Pacific, Morskoi Gidrof. Zh. (Marine Hydrophysical Jowrnal),4,59-61, 1988, (in Russian).

Serebryany, A., Nonlinear effects in internal waves on a shelf, Izvestiya, Atmospheric and Oeeanic Physics,26, 285 - 293, 1990.

Shrira, V., On the long strongly nonlinear waves in rotating ocean, Izvestiya, Atmospheric and Oceanic Physics,22, 395 $405,1986$.

Smyth, N. F., and Holloway, P. E., Hydraulic jump and undular bore formation on a Shelf Break, J. Phys. Oceanogr.,18, 947 $962,1988$.

Zheng, Q., Yan, X. H., and Klernas, V., Statistical and dynamical analysis of internal waves on the continental ahelf of the Middie Atlantic Bight from opace whuttle photographe, J. Geophys. Res.,C98, 8495 - 8504, 1993.

Zhou, X., Grimalhaw, R., The effect of variable currents on internal colitary waves, Dyn. Atm. Oceans,14, 17 - 39, 1989. 